\title{
High-mobility group box-1 protein induces osteogenic phenotype changes in aortic valve interstitial cells
}

\author{
Bo Wang, PhD, ${ }^{\mathrm{a}}$ Fei Li, MD, ${ }^{\mathrm{a}}$ Chao Zhang, $\mathrm{PhD},{ }^{\mathrm{a}}$ Guangxia Wei, MD, ${ }^{\mathrm{c}}$ Pingping Liao, $\mathrm{PhD},{ }^{\mathrm{b}}$ and \\ Nianguo Dong, $\mathrm{MD}, \mathrm{PhD}^{\mathrm{a}}$
}

\begin{abstract}
Objectives: Calcific aortic valve (AV) disease is known to be an inflammationrelated process. High-mobility group box-1 (HMGB1) protein and Toll-like receptor 4 (TLR4) have been reported to participate in several inflammatory diseases. The purpose of the present study was to determine whether the HMGB1-TLR4 axis is involved in calcific AV disease, and to evaluate the effect of HMGB1, and its potential mechanisms, on the pro-osteogenic phenotype change of valvular interstitial cells (VICs).
\end{abstract}

Methods: Expression of HMGB1 and TLR4 in human calcific AVs was evaluated using immunohistochemical staining and immunoblotting. Cultured VICs were used as an in vitro model. The VICs were stimulated with HMGB1 for analysis, with versus without TLR4 small interfering ribonucleic acid (siRNA), c-Jun $\mathrm{N}$-terminal kinase mitogen-activated protein kinase (JNK MAPK), and nuclear factor kappa-B $(\mathrm{NF}-\kappa \mathrm{B})$ inhibitors.

Results: Enhanced accumulation of HMGB1 and TLR4 was observed in calcific valves. Moreover, we found that HMGB1 induced high levels of proinflammatory cytokine production and promoted the osteoblastic differentiation and calcification of VICs. In addition, HMGB1 induced phosphorylation of JNK MAPK and NF- $\kappa$ B. However, these effects were markedly suppressed by siRNA silencing of TLR4. In addition, blockade of JNK MAPK and NF- $\kappa$ B phosphorylation prohibited HMGB1-induced production of pro-osteogenic factors, and mineralization of VICs.

Conclusions: The HMGB1 protein may promote osteoblastic differentiation and calcification of VICs, through the TLR4-JNK-NF- $\kappa$ B signaling pathway. (J Thorac Cardiovasc Surg 2016;151:255-62)

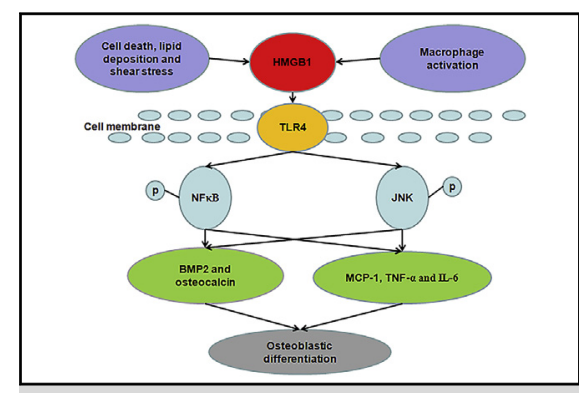

The HMGB1 protein may promote the osteoblastic differentiation of VICs, through the TLR4 pathway.

\section{Central Message}

The HMGB1 protein promotes AV calcification through a pro-inflammatory effect, and might be a new target for therapeutic intervention.

\section{Perspective}

The identification of TLR4 signaling as being downstream of HMGB1 provides novel insight into the function of the HMGB1-TLR4 pathway in the pathogenesis of $\mathrm{AV}$ calcification in vitro. Identification of the causal relationship between HMGB1 and calcific AV disease might reveal that HMGB1 might serve as a target to predict and prevent calcific AV disease.

See Editorial Commentary page 263.
Calcific aortic valve (AV) disease is a common health problem associated with high mortality and morbidity in aging societies in which the average age is increasing.

\footnotetext{
From the ${ }^{\mathrm{a}}$ Department of Cardiovascular Surgery, Union Hospital, Tongji Medical College, Huazhong University of Science and Technology, Wuhan, China; ${ }^{\mathrm{b}} \mathrm{De}-$ partment of Gerontology, Union Hospital, Tongji Medical College, Huazhong University of Science and Technology, Wuhan, China; and ${ }^{\mathrm{c}}$ Department of Cardiothoracic Surgery, Huangshi Central Hospital, Huangshi, China.

B.W. and F.L. are co-first authors.

This work was supported by the National Natural Science Foundation of China (81300175 and 81270297).

Received for publication July 21, 2015; revisions received Sept 14, 2015; accepted for publication Sept 14, 2015; available ahead of print Oct 26, 2015.

Address for reprints: Nianguo Dong, MD, PhD, Department of Cardiovascular Surgery, Union Hospital, Tongji Medical College, Huazhong University of Science and Technology, 1277 Jiefang Ave, Wuhan 430022, China (E-mail: dongnianguo@ hotmail.com).

$0022-5223 / \$ 36.00$

Copyright (C) 2016 by The American Association for Thoracic Surgery

http://dx.doi.org/10.1016/j.jtcvs.2015.09.077
}

Historically, calcific AV disease was considered to be a passive process. Recent studies indicate that calcific AV disease is an active disease that has several pathogeneses that are similar to vascular calcification, such as inflammation, osteogenesis, and mineralization. ${ }^{1,2}$ Currently, the only effective therapy is valve replacement or implantation, yet not everyone is appropriate for or can tolerate this surgery.

The high-mobility group box 1 (HMGB1) is a ubiquitous nuclear protein that has cytokine, chemokine, and growth-factor activity, orchestrating the inflammatory and immune response in mammals. This protein has been reported to be involved in the pathogenesis of vascular atherosclerosis and inflammation changes, 4-7 $^{-7}$ and it facilitates the assembly of nuclear proteins and participates in deoxyribonucleic acid (DNA) replication, recombination, transcription, and repair. ${ }^{8}$ However, 


\section{Abbreviations and Acronyms \\ $\mathrm{AV}=$ aortic valve \\ BMP2 = bone morphogenetic protein 2 \\ HMGB1 = high-mobility group box-1 protein \\ JNK MAPK $=$ c-Jun N-terminal kinase mitogen- activated protein kinase \\ $\mathrm{NF}-\kappa \mathrm{B}=$ nuclear factor kappa-B \\ siRNA $=$ small interfering ribonucleic acid \\ TLR $=$ Toll-like receptor \\ TNF- $\alpha=$ tumor necrosis factor- $\alpha$ \\ VIC $=$ valvular interstitial cell}

whether HMGB1 plays a role in calcific AV disease is unknown.

Pro-osteogenic differentiation of valvular interstitial cells (VICs) is considered to be a key cellular mechanism in the development of AV calcification. Studies indicate that human VICs that are isolated from calcific AV leaflets express more Toll-like receptor 4 (TLR4); and activating TLR4 results in the osteoblastic differentiation of VICs. ${ }^{9}$ In addition, HMGB 1 is one of the important agonists of TLR4. ${ }^{10-14}$ However, little is known about the association between HMGB1 and TLR4 in the pathogenesis of AV calcification.

\section{METHODS}

\section{Chemicals and Reagents}

The following antibodies were used: HMGB1, osteocalcin, and bone morphogenetic protein (BMP)2 (Abcam, Cambridge, Mass); TLR4 (Santa Cruz Biotechnology, Santa Cruz, Calif); and phosphorylated and total NF- $\kappa$ B and JNK MAPK (Cell Signaling Technology, Danvers, Mass). Beta-actin was used as a normalization for total and cytosolic protein determination. Recombinant HMGB1 (R\&D Systems, Minneapolis, Minn) and collagenase and other reagents (Sigma-Aldrich, St Louis, Mo) were used as well.

\section{Calcific Aortic Valve Collection}

This study complied with the Declaration of Helsinki and was approved by the review boards of Union Hospital and Tongji Medical College; all patients provided written informed consent. Calcific AV leaflets were obtained intraoperatively from 15 patients undergoing $\mathrm{AV}$ replacement in Union Hospital to treat severe AV calcification. The thin and "normal" AV leaflets were collected from 10 age-matched patients undergoing Bentall surgery to treat acute aortic dissection. Demographic information for patients is summarized in Table 1. The tissue samples were kept frozen in liquid nitrogen until use. All valves were tricuspid.

\section{Cell Culture and Treatment}

Valvular interstitial cells were isolated from patients undergoing Bentall surgery for acute aortic dissection, using the collagenase I digestion method previously described. ${ }^{15}$ Briefly, isolated leaflets were digested in essential medium containing $1 \mathrm{mg} / \mathrm{ml}$ of collagenase type I at $37^{\circ} \mathrm{C}$ for 30 minutes. After removal of endothelial cells by vortexing, the leaflets were further digested with a fresh solution of $1 \mathrm{mg} / \mathrm{ml}$ of collagenase medium for 4 to 6 hours at $37^{\circ} \mathrm{C}$. After vortexing and repeated aspirating to break up the tissue mass, the suspension was spun at $1000 \mathrm{rpm}$ for 10 minutes to precipitate cells. Cells were resuspended and cultured in essential medium, supplemented with $100 \mathrm{U} / \mathrm{ml}$ penicillin, $100 \mu \mathrm{g} / \mathrm{ml}$ streptomycin, and $10 \%$ fetal bovine serum, in an incubator with $5 \% \mathrm{CO}_{2}$, at $37^{\circ} \mathrm{C}$. Cells of passages 2 to 5 were used for all experiments. When they had grown to $70 \%$ to $90 \%$ confluence, the VICs were stimulated with HMGB 1 in the presence of the condition medium: $10 \mathrm{mmol}$ of $\beta$-glycerophosphate; $100 \mathrm{nmol}$ of dexamethasone; and $50 \mu \mathrm{g} / \mathrm{ml}$ ascorbic acid (all 3 from Sigma-Aldrich, St Louis, Mo). If needed, pharmacologic reagents, including $10 \mu \mathrm{mol} / \mathrm{L}$ SP600125 and $10 \mu \mathrm{mol} / \mathrm{L}$ BAY 11-7082 (both from Beyotime, Nantong, People's Republic of China), were added 1 hour before the addition of HMGB1. Cells from 3 patients were used for each intervention.

\section{Real-Time Polymerase Chain Reaction RNA Analysis}

Real-time polymerase chain reaction analysis was used to detect expression of messenger RNAs encoding interleukin-6, tumor necrosis factor- $\alpha$, and monocyte chemoattractant protein-1. The RNA was isolated and reversely transcribed to complementary DNA (deoxyribonucleic acid), as previously described. ${ }^{15}$ Real-time polymerase chain reaction assays were carried out using an SYBR Premix Ex Taq (Takara Bio Inc, Otsu, Japan) on a StepOnePlus Real-Time PCR System (Applied Biosystems, Foster City, Calif). Primers were as follows: interleukin-6 (F: 5'-ATC AGG AGA CCT GCT TGA TG-3'; R: 5'-TGG TGG CTT TGT CTG GAT TC-3'); tumor necrosis factor- $\alpha$ (F: $5^{\prime}$-CCA ATG GCA GAG TGG GTA TG-3'; R: $5^{\prime}$-TGA AGA GGA CCT GGG AGT AG-3'); monocyte chemoattractant protein-1 (F: 5'-GTC ACC AGC AGC AAG TGT C-3'; R: 5'CCA GGT GGC TTA TGG AGT C- $3^{\prime}$ ); and $\beta$-actin (F: $5^{\prime}$-GAC CTG ACC GAC TAC CTC-3'; R: 5'-GCT TCT CCT TGATGT CCC-3'). Results were normalized to $\beta$-actin expression and analyzed by the comparative $\mathrm{C}_{\mathrm{T}}$ (real-time polymerase chain reaction data) method.

\section{Silencing Toll-like Receptor 4}

To knock down TLR4, cells (60\%-80\% confluence) in 6-well plates were incubated with a mixture of siRNA $(50 \mathrm{nmol} / \mathrm{L})$, and control cells were treated with scrambled siRNA using Lipofectamine 2000 (Invitrogen, Carlsbad, Calif) and Opti-MEM (Life Technologies, Carlsbad, Calif) according to manufacturer instructions. The medium was changed 4 to 6 hours after transfection; 72 hours later, the cells were harvested for protein expression analysis.

\section{Alizarin Red Staining}

For mineralization experiments, aortic VICs were seeded in 24-well plates. After $70 \%$ confluence was achieved, cells were incubated, per indicated interventions, in essential medium supplemented with $5 \%$ fetal bovine serum, $2 \mathrm{mmol} / \mathrm{L} \beta$-glycerophosphate, $100 \mathrm{nmol} / \mathrm{L}$ dexamethasone, and $50 \mu \mathrm{g} / \mathrm{ml}$ ascorbic acid for 14 days. Alizarin red staining for calcium deposits was performed as described previously. ${ }^{16}$ Briefly, cell monolayers were washed twice with phosphate-buffered saline and fixed for 15 minutes in $4 \%$ paraformaldehyde. After incubation with $0.2 \%$ alizarin red solution ( $\mathrm{pH}$ : 4.2) for 30 minutes, excessive dye was removed, by washing with distilled water. To quantify the staining, cell monolayers were bleached with $10 \%$ acetic acid at $75^{\circ} \mathrm{C}$. Supernatant was spectrophotometrically analyzed at $450 \mathrm{~nm}$.

\section{Immunohistochemistry}

Immunohistochemical detection of HMGB1 protein in AV leaflets was performed by using the biotin-linked peroxidase technique. Paraffin 
TABLE 1. Clinical characteristics in patients who have calcific AV stenosis, versus control subjects

\begin{tabular}{lcc}
\hline \multicolumn{1}{c}{ Characteristics } & $\begin{array}{c}\text { Patients with } \\
\text { calcific AV stenosis }\end{array}$ & $\begin{array}{c}\text { Control } \\
\text { subjects }\end{array}$ \\
\hline Patients (n) & 15 & 10 \\
Age (y) & $60.6 \pm 4.2$ & $58.4 \pm 5.7$ \\
Gender, male & $11(73.3)$ & $8(80)$ \\
Body mass index $\left(\mathrm{kg} / \mathrm{m}^{2}\right)$ & $23.8 \pm 3.5$ & $23.1 \pm 4.3$ \\
Risk factors & & \\
$\quad$ Hypertension & $8(53.3)$ & $4(40)$ \\
Hypercholesterolemia & $11(73.3)$ & $4(40)$ \\
Diabetes mellitus & $3(20)$ & $1(10)$ \\
$\quad$ Smoking & $11(73.3)$ & $8(80)$ \\
Medications & $6(40)$ & $3(30)$ \\
$\quad$ Statins & $8(53.3)$ & $3(40)$ \\
ACEi/ARB & $8(53.3)$ & $2(20)$ \\
$\quad \beta$-blockers & & \\
Echocardiographic parameters & $59.3 \pm 5.7$ & $53.1 \pm 6.1$ \\
LVEF (\%) & $82.7 \pm 7.1$ & $16.3 \pm 6.5$ \\
Transvalvular gradient (mm Hg) & $0.7 \pm 0.1$ & $3.2 \pm 0.3$ \\
Aortic valve area $\left(\mathrm{cm}^{2}\right)$ & $10(66.7)$ & $8(80)$ \\
Aortic insufficiency &
\end{tabular}

Values are $\mathrm{n}(\%)$, or mean $\pm \mathrm{SD}$, unless otherwise indicated. For the difference between groups for transvalvular gradient and aortic valve area, $P<.05$. All other $P$ values were nonsignificant. $A V$, Aortic valve; $A C E i$, angiotensin-converting enzyme inhibitor; $A R B$, angiotensin receptor blocker; $L V E F$, left ventricular ejection fraction.

sections $(5 \mathrm{~mm})$ were prepared and dried at room temperature for 2 hours. Immunohistochemical staining was performed in the AV leaflets as previously described. ${ }^{15}$ The sections were incubated with antibodies against HMGB1 (1:300) and TLR4 (1:200). Image-Pro Plus (Media Cybernetics, Inc, Bethesda, Md) was used to determine the quantitative results.

\section{Immunoblotting}

Tissue samples and cells were homogenized and Western blotting was performed as previously described. ${ }^{15}$ The following primary antibodies were used: HMGB1 (1:1000 dilution); TLR4 (1:200 dilution); BMP2 (1:1000 dilution); osteocalcin (1:1000 dilution); phospho-JNK MAPK (1:500 dilution); total JNK MAPK (1:1000 dilution); phospho-NF- $\kappa$ B (1:500 dilution), and total NF- $\kappa \mathrm{B}$ (1:1000 dilution). Beta-actin was used as a normalization medium for total versus cytosolic protein determination. In the phosphorylation induction assay, indicated total protein expression levels were used for normalization. Bands were quantified by densitometry, using Quantity-One Software (Bio-Rad Laboratories, Inc, Hercules, Calif). In the phosphorylation induction assay, the indicated total protein expression was used for normalization.

\section{Statistical Analysis}

All data were analyzed using SPSS, version 13.0 (SPSS, Inc, Chicago, Ill). All values are presented as mean \pm SE. Univariate analyses were performed using $\chi^{2}$ analysis for categoric variables. After confirming that all variables were normally distributed, using the Kolmogorov-Smirnov test, the Student $t$-test was applied to analyze data comparisons between normal and calcific aortic valves; a 1-way analysis of variance was used to compare differences between experimental groups.

\section{RESULTS}

\section{Expression of HMGB1 and TLR4 in Calcific AV Leaflets}

Previous studies have demonstrated that HMGB1 and TLR4 participate in several cardiovascular diseases. ${ }^{5,6,8}$
In the present study, we used immunohistochemical staining and immunoblotting to determine whether HMGB1 and TLR4 are involved in calcific AV disease (Figure 1, A-D). We found that the expression of HMGB 1 and TLR4 were markedly increased in calcific AVs, compared to normal ones (by 1.94-fold and 1.73-fold, respectively; Figure 1, $E$ and $F$ ). These results suggest that HMGB1 and TLR4 may play a role in the development of $\mathrm{AV}$ calcification.

\section{The HMGB1-TLR4 Axis Induces Osteogenic Differentiation and Calcification of VICs}

Previous studies demonstrated that HMGB1 may be an endogenous ligand of TLR4, in several cell types. ${ }^{10,11}$ This finding led us to hypothesize that HMGB1-induced TLR4 activation trigger the intracellular signaling that results in the osteoblastic differentiation of VICs. To evaluate this possibility, we incubated VICs with HMGB $1(0,0.01,0.1$, and $1 \mu \mathrm{g} / \mathrm{ml})$ for 72 hours. We examined the expression of TLR4 and 2 typical proosteogenic markers: bone morphogenetic protein 2 (BMP2) and osteocalcin. We found that TLR4, BMP2, and osteocalcin levels increased significantly in a dosedependent manner with increasing concentrations of HMGB1 (Figure 2, $A-C$ ).

To investigate the causal relationship between TLR4 activation and HMGB1-induced osteoblastic differentiation of VICs, we used specific siRNAs to target human TLR4. We found that TLR4 siRNA efficiently and specifically knocked down the expression of TLR4 (Figure 2, $D$ ). In addition, pretreatment with TLR4 siRNA markedly suppressed the up-regulatory effect of HMGB1 on BMP2 and osteocalcin, as well as mineralization (Figure 2,E and $F)$. Collectively, our data suggest that activation of TLR4 mediates the HMGB1-induced osteoblastic differentiation and mineralization of VICs (Figure 6).

\section{Inflammatory Responses in VICs Through TLR4}

The role of TLR4 in mediating macrophage activation and pro-inflammatory cytokine production has been well characterized. ${ }^{10,14}$ Hence, we further explored whether HMGB1 could induce inflammatory response via the TLR4 pathway in human VICs. We stimulated VICs with HMGB $1(1 \mu \mathrm{g} / \mathrm{ml})$, and measured inflammatory cytokine levels after 24 hours. Under basal conditions, cultured VICs released low levels of monocyte chemoattractant protein-1, tumor necrosis factor (TNF)- $\alpha$, and interleukin (IL)-6. However, treatment with HMGB1 significantly augmented the production of monocyte chemoattractant protein-1, TNF- $\alpha$, and interleukin-6 (by 3.97-fold, 4.03fold and 2.80-fold, respectively). This augmentation was blocked when TLR4 expression was inhibited by TLR4 siRNA silencing (Figure 3, $A-C$ ). 


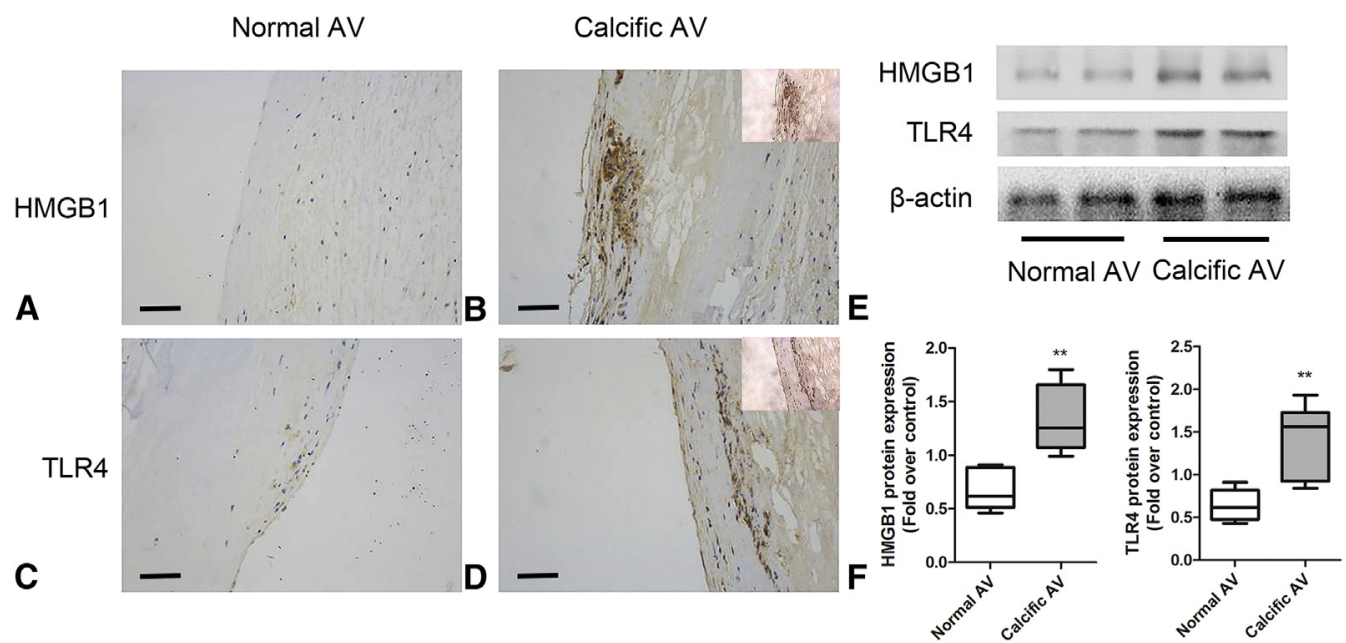

FIGURE 1. The expression of HMGB1 and TLR4 is increased in human calcific AVs. A-D, Representative images of immunohistochemical staining of HMGB1 and TLR4 in human calcific AVs and normal AVs. E and F, Immunoblotting showed that HMGB1 and TLR4 were significantly increased in human calcific $\mathrm{AVs}$ (healthy AVs, $\mathrm{n}=10$; calcific $\mathrm{AVs}, \mathrm{n}=15 ; A V$, Aortic valve; $H M G B 1$, high-mobility group box-1 protein; TLR4, Toll-like receptor 4 . ** $P<.01$.

\section{Phosphorylation of NF- $\kappa$ B and JNK MAPK in a \\ TLR4-Dependent Manner Is Induced by HMGB1}

Evidence shows that HMGB1 can induce activation of the $\mathrm{NF}-\kappa \mathrm{B}$ and JNK MAPK pathways in various cells. ${ }^{12-14}$ We tested whether TLR4 is required for HMGB1-induced
NF- $\kappa$ B and JNK MAPK phosphorylation in VICs. The VICs were pretreated with TLR4 siRNA and stimulated with $1 \mu \mathrm{g} / \mathrm{ml}$ HMGB 1 for 1 hour. We found that HMGB 1 induced the phosphorylation of NF- $\kappa \mathrm{B}$ and JNK MAPK (by 2.67- and 2.81-fold, respectively). However,

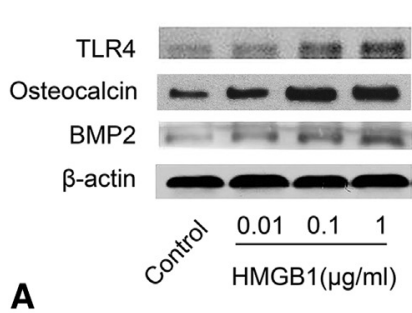

A

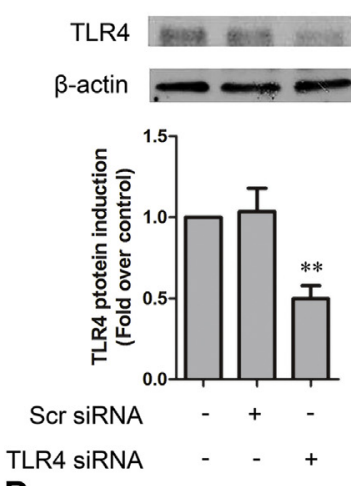

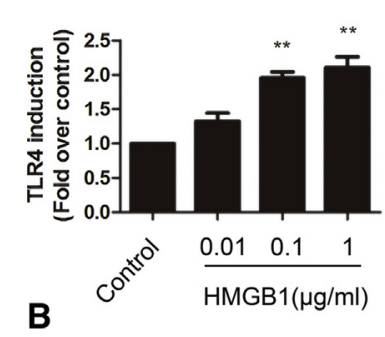

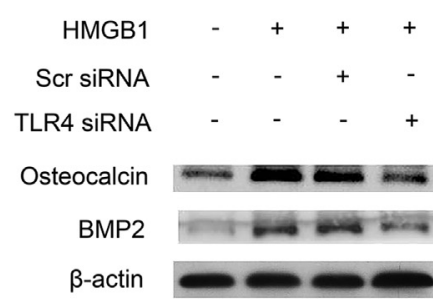

E
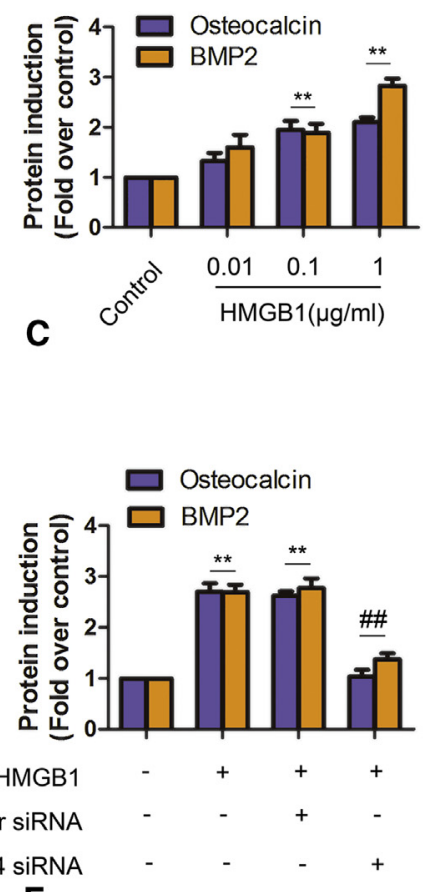

$\mathbf{F}$

FIGURE 2. The HMGB1 protein induced osteoblastic differentiation of aortic valve interstitial cells, via TLR4. A-C, The HMGB1 treatment increased the expression of TLR4, BMP2, and osteocalcin in a dose-dependent manner. D-F, The TLR4 siRNA significantly (D) reduced the expression of TLR4; and (E and F) suppressed the up-regulation of BMP2 and osteocalcin induced by HMGB1. Data are presented as the mean \pm SE (error bars) of independent experiments in cells from 3 different aortic valve tissues. TLR4, Toll-like receptor 4; BMP2, bone morphogenetic protein 2; HMGB1, high-mobility group box-1 protein; Scr siRNA, scrambled small interfering ribonucleic acid. ${ }^{* *} P<.01$ versus control subjects. \#\#P $<.01$ versus HMGB1 + Scr siRNA. 


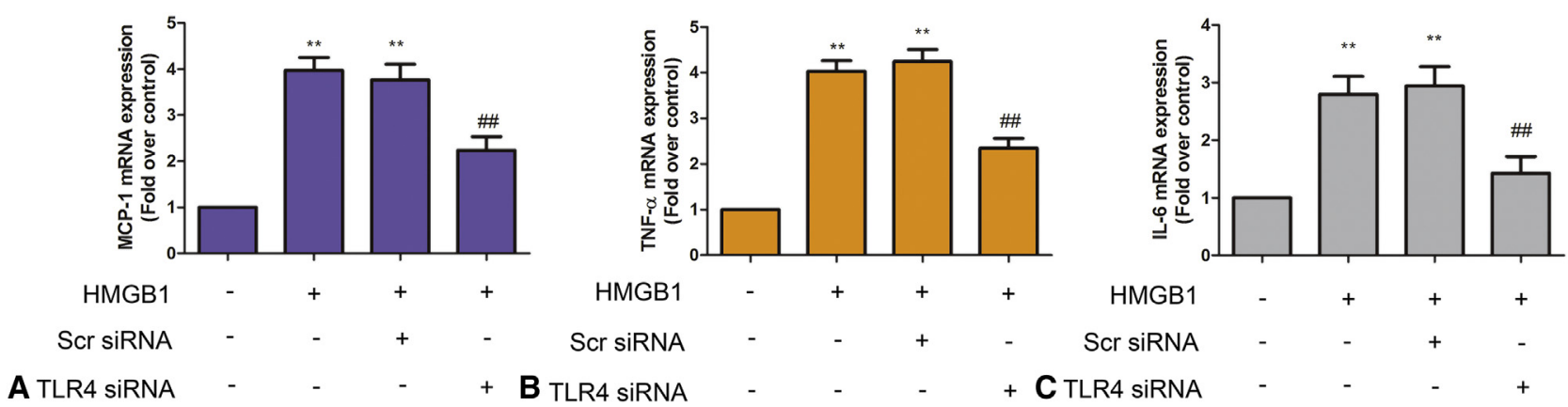

FIGURE 3. Quantitative polymerase chain reaction analysis of inflammatory cytokine mRNA expression in valvular interstitial cells. The HMGB1 markedly augmented mRNA expression of (A) MCP-1, (B) TNF- $\alpha$, and (C) IL-6, which was attenuated by TLR4 siRNA. Data are presented as the mean \pm SE (error bars) of independent experiments in cells from 3 different aortic valve tissues. $M C P$, Monocyte chemoattractant protein; $m R N A$, messenger RNA; $T N F$, tumor necrosis factor; $I L$, interleukin; HMGB1, high-mobility group box; Scr siRNA, scrambled small interfering ribonucleic acid; TLR4, Toll-like receptor 4. ${ }^{* *} P<.01$ versus control. \#\#P<.01 versus HMGB1 + Scr siRNA.

pretreatment with TLR4 siRNA inhibited the phosphorylation of NF- $\kappa$ B and JNK MAPK (Figure 4, $A$ and $B$ ). These data clearly demonstrated that HMGB1 induced the phosphorylation of NF- $\kappa$ B and JNK MAPK in VICs, likely through a TLR4-dependent pathway.

We used Bay 11-7082 (a NF- $\kappa$ B inhibitor) and SP600125 (a JNK MAPK inhibitor) to confirm whether NF- $\kappa$ B and JNK MAPK signaling functionally mediates HMGB1induced osteoblastic differentiation of VICs. In the presence of Bay 11-7082 and SP600125, HMGB1-induced elevation of BMP2 and osteocalcin was significantly inhibited (Figure $4, C$ ). Moreover, inhibition of NF- $\kappa \mathrm{B}$ and JNK MAPK attenuated the calcium deposits of VICs (Figure 5, $A$ and $B$ ). Taken together, these data demonstrated that the
TLR4-dependent NF- $\kappa \mathrm{B}$ and JNK MAPK signaling pathway mediated the osteoblastic differentiation and calcification of VICs induced by HMGB1.

\section{DISCUSSION}

In the present study, we found that expression of HMGB1 and TLR4 in calcific AVs was higher than that in normal valves. In cultured human aortic VICs, HMGB1 induced the up-regulation of BMP2 and osteocalcin. Additionally, HMGB1 augmented the production of the pro-inflammatory cytokines monocyte chemoattractant protein-1, TNF- $\alpha$, and interleukin-6. All these effects were mediated by TLR4 activation. Furthermore, the NF$\kappa \mathrm{B}$ and JNK MAPK pathways are involved in the signaling

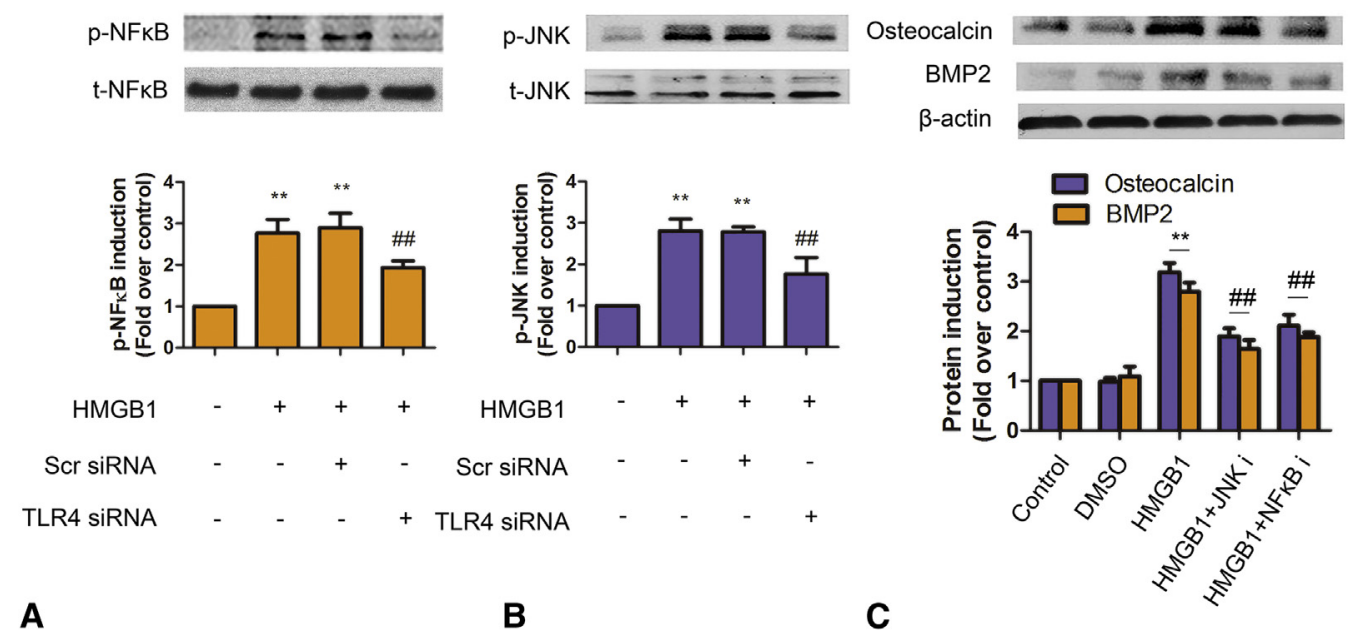

FIGURE 4. The HMGB1-induced osteoblastic differentiation of valve interstitial cells, which is TLR4 dependent, and mediated by NF- $\kappa \mathrm{B}$ and JNK MAPK. A and B, The HMGB1 protein induced the phosphorylation of NF- $\kappa$ B and JNK MAPK, which was suppressed by TLR4 silencing. C, In the presence of NF- $\kappa$ B and JNK MAPK inhibitors, the HMGB1-up-regulated expression of both BMP2 and osteocalcin were decreased significantly. Data are presented as the mean $\pm \mathrm{SE}$ (error bars) of independent experiments in cells from 3 different aortic valve tissues. $N F-\kappa B$, Nuclear factor kappa-B; $J N K$, c-Jun $\mathrm{N}$-terminal kinase; $B M P$, bone morphogenetic protein; HMGB1, high-mobility group box; Scr siRNA, scrambled small interfering ribonucleic acid; TLR4 siRNA, Toll-like receptor 4 small interfering ribonucleic acid; $D M S O$, dimethyl sulfoxide. $* * P<.01$ versus control. \#\#P<.01 versus HMGB1 + Scr siRNA or HMGB1. 


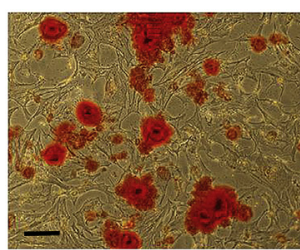

Control

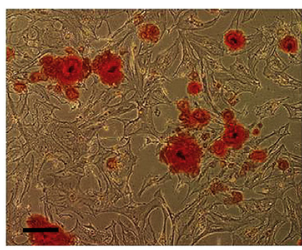

HMGB1+JNK i

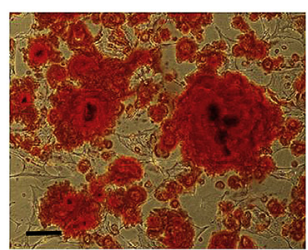

HMGB1

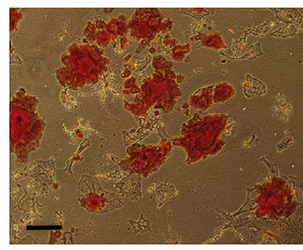

HMGB1+NFKB i

A

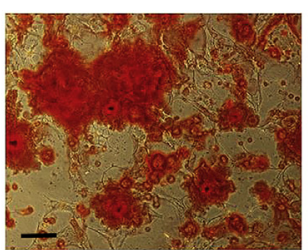

HMGB1+Scrambled siRNA

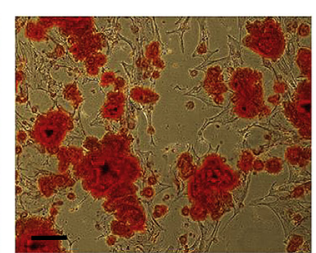

HMGB1+TLR4 siRNA

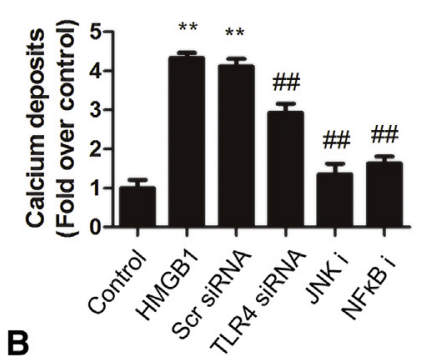

FIGURE 5. Analysis of calcium deposition of VICs induced by different treatment. A, Representative alizarin red staining of VICs. Scale bar $=200 \mu \mathrm{m}$. B, Quantitative analysis showed that HMGB1 increased calcium deposition in VICs; TLR4 siRNA, NF- $\kappa$ B, and JNK MAPK inhibitors attenuated this effect. Data are presented as the mean $\pm \mathrm{SE}$ (error bars) of independent experiments in cells from 3 different aortic valve tissues. $H M G B 1$, High-mobility group box; siRNA, small interfering ribonucleic acid; TLR4, Toll-like receptor $4 ; J N K$, c-Jun N-terminal kinase; $N F-\kappa B$, nuclear factor kappa-B. ** $P<.01$ versus control. \#\#P<.01 versus HMGB1 + Scr siRNA or HMGB1.

mechanism for the osteoblastic differentiation of VICs that is induced by the HMGB1-TLR4 axis. These data offer novel insight into the role of the HMGB1-TLR4 axis in the pathogenesis of $\mathrm{AV}$ calcification.

The HMGB1 protein is the most highly expressed of all the HMG family members ${ }^{17}$ and plays a critical role not only inside the cell, as a DNA chaperone, and chromosome

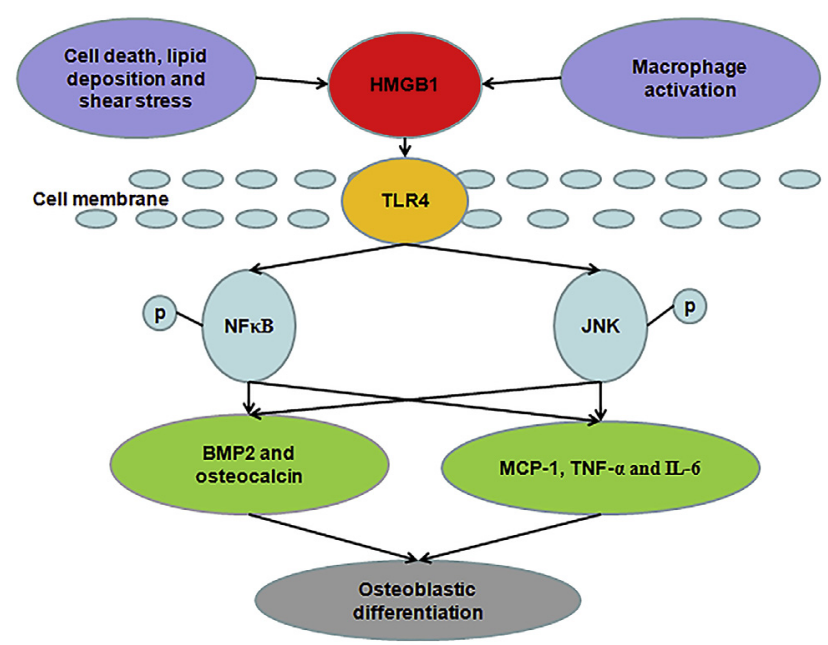

FIGURE 6. Schematic illustration of the role of (HMGB1 and TLR4 in the development of osteoblastic phenotype change in human aortic valve interstitial cells. $H M G B 1$, High-mobility group box 1; TLR4, Toll-like receptor $4 ; p$, phosphorylated; $N F-\kappa B$, nuclear factor kappa-B; $J N K$, c-Jun N-terminal kinase; $B M P$, bone morphogenetic protein; $M C P$, monocyte chemoattractant protein; $T N F$, tumor necrosis factor; $I L$, interleukin. guardian, but also outside the cell as the prototypic damageassociated molecular pattern molecule. Several studies have found that HMGB1 acts as a pro-inflammatory mediator in vascular injury and atherosclerosis. ${ }^{4,5}$ In atherosclerotic disease, HMGB1 displays increased expression in nuclei and the cytoplasm of macrophages and smooth muscle cells, and it is implicated in the progression of atherosclerotic plaque. In this study, we additionally found that HMGB1 was up-regulated in calcific AVs, which is perhaps the first evidence showing the involvement of HMGB1 in calcific AV disease.

Toll-like receptor 4 is a member of the Toll-like receptors superfamily, which can recognize not only pathogen-associated molecular patterns, but also endogenous ligands or damage-associated molecular patterns. Several lines of evidence demonstrate that TLR4 activation induces osteoblastic differentiation and inflammation in aortic VICs. ${ }^{9,18,19}$ We therefore speculated that HMGB1 induces the pro-osteogenic phenotype change of VICs, via TLR4 activation. Expression of TLR4, BMP2, and osteocalcin were assessed in cultured VICs after stimulation with HMGB1. Bone morphogenetic protein 2 is an osteoinductive factor that promotes osteogenic differentiation in a variety of cells, and osteocalcin is a typical calcification marker. ${ }^{20}$ In this study, the expression of TLR4, BMP2, and osteocalcin was up-regulated after treatment with HMGB1 in a dose-dependent manner. The introduction of siRNA to knock down TLR4 expression blunted this effect, suggesting that TLR4 mediated the HMGB1-induced osteoblastic differentiation of VICs. 
Observations and experimental evidence strongly suggest that TLR4 signaling results in profound inflammation. $^{9,21}$ Our in vitro data demonstrated that HMGB1 activated TLR4 and induced subsequent signaling pathways in VICs, resulting in the increased production of the pro-inflammatory cytokines interleukin- 6 , TNF- $\alpha$, and monocyte chemoattractant protein-1. The role of interleukin-6 in inflammation-induced calcification is well established. ${ }^{22}$ Monocyte chemoattractant protein-1 has been associated with macrophage infiltration, in a rabbit model of AV calcification. ${ }^{23}$ Tumor necrosis factor- $\alpha$ may be a critical downstream mediator of inflammation-induced calcification, through the BMP2Dlx5 pathway, and the TNF- $\alpha$ neutralizing antibody, known as infliximab, inhibits the aortic Msx-Wnt calcification cascade in Ldlr-/- mice. ${ }^{24,25}$ In addition, we observed that TLR4 siRNA suppressed HMGB1stimulated up-regulation of these cytokines. We may conclude that the blockade of the TLR4 pathway alleviated the pro-inflammatory effect of HMGB1 on VICs. These data suggested that inflammation seems to be a key mediator between HMGB1-induced TLR4 activation and $\mathrm{AV}$ calcification.

The activation of TLR4 relays cell surface signals to multiple intracellular pathways, including p38 MAPK, JNK MAPK, and NF- $\kappa$ B. ${ }^{26-28}$ In a previous study, we demonstrated that JNK MAPK mediates ox-low-density lipoprotein-induced osteoblastic differentiation in VICs. ${ }^{16}$ The NF- $\kappa$ B pathway is a canonical pathway responsible for the production of pro-inflammatory cytokines, which are associated with osteoblastic differentiation. ${ }^{19,29,30}$ High-glucose-induced HMGB1 activates NF- $\kappa \mathrm{B}$, which is involved in mediating the expression of BMP2 in smooth muscle cells. ${ }^{31}$

In the present study, HMGB1 activated the NF- $\kappa \mathrm{B}$ and JNK MAPK signaling pathway in a TLR4-dependent manner. Furthermore, blockade of the NF- $\kappa \mathrm{B}$ and JNK MAPK signaling pathways significantly prohibited the up-regulation of BMP2 and osteocalcin and calcium deposits induced by HMGB1 (Figure 6). However, the relationship between the NF- $\kappa \mathrm{B}$ and JNK MAPK pathways has not been investigated in this study. Our results expand on those of the previous study and indicate that NF- $\kappa \mathrm{B}$ and JNK MAPK activation mediates HMGB1-induced osteoblastic differentiation of VICs.

To our knowledge, no pharmacologic therapies can modify the progression of aortic stenosis. Statins were previously first-line candidates for slowing progression of the disease. Despite promising animal experiments, ${ }^{32}$ several clinical trials showed that statins failed to alter the progression of aortic stenosis. ${ }^{33,34}$ We demonstrated the role of HMGB1 in vitro, with emphasis on its pro-inflammation effect, but it may have other effects. This protein could be a new target for therapeutic intervention. A recent study reported that treatment with HMGB1 neutralizing antibody reduces development of diet-induced atherosclerosis in $\mathrm{ApoE}^{-1-}$ mice. ${ }^{4}$ In addition, the HMGB1 monoclonal antibody may exert a beneficial effect in modulating the progression of aortic stenosis. However, much work is needed in this area before results will be able to directly assist patients.

\section{Limitations}

This study has limitations. First, HMGB1 has been reported to be an agonist of TLR2 and a receptor for advanced glycation end products in several cell types. In the present study, we identified the association between the HMGB1-TLR4 axis and VICs calcification in vitro. However, signaling pathways might communicate or cross-talk in regulating the cellular response to HMGB1. We cannot exclude the possibility that HMGB1 might induce VIC calcification via other pathways. Second, the mechanism underlying the source of HMGB1 during AV calcification development was not investigated in the study. Additional work is required to explore the causal relationship between HMGB1 and inflammation in calcific $\mathrm{AV}$ disease.

In summary, we examined the molecular association between HMGB1 and TLR4 in the osteoblastic differentiation of VICs. Our results show that HMGB1 induced TLR4dependent pro-osteogenic and pro-inflammatory factors expression, which lead to the osteoblastic transformation and mineralization of VICs. The identification of TLR4 signaling as being downstream of HMGB1 provides a novel insight into the function of the HMGB1-TLR4 axis in the pathogenesis of $\mathrm{AV}$ calcification in vitro.

\section{Conflict of Interest Statement}

Authors have nothing to disclose with regard to commercial support.

\section{References}

1. Rajamannan NM. Calcific aortic stenosis lessons learned from experimental and clinical studies. Arterioscler Thromb Vasc Biol. 2009;29:162-8.

2. Miller JD, Weiss RM, Heistad DD. Calcific aortic valve stenosis: methods, models, and mechanisms. Circ Res. 2011;108:1392-412.

3. Agarwal S, Garg A, Parashar A, Svensson LG, Tuzcu EM, Navia JL, et al. In-hospital mortality and stroke after surgical aortic valve replacement: a nationwide perspective. J Thorac Cardiovasc Surg. 2015;150:571-8.

4. Kanellakis P, Agrotis A, Kyaw TS, Koulis C, Ahrens I, Mori S, et al. Highmobility group box protein 1 neutralization reduces development of diet induced atherosclerosis in apolipoprotein e-deficient mice. Arterioscler Thromb Vasc Biol. 2011;31:313-9.

5. de Souza AW, Westra J, Limburg PC, Bijl M, Kallenberg CG. HMGB1 in vascular diseases: its role in vascular inflammation and atherosclerosis. Autoimmunol Rev. 2012;11:909-17.

6. Hashimoto T, Ishii J, Kitagawa F, Yamada S, Hattori K, Okumura M, et al. Circulating high-mobility group box 1 and cardiovascular mortality in unstable angina and non-ST-segment elevation myocardial infarction. Atherosclerosis. 2012;221 490-5.

7. Luo Y, Li SJ, Yang J, Qiu YZ, Chen FP. HMGB1 induces an inflammatory response in endothelial cells via the RAGE-dependent endoplasmic reticulum stress pathway. Biochem Biophys Res Commun. 2013;438:732-8. 
8. Andrassy M, Volz HC, Igwe JC, Funke B, Eichberger SN, Kaya Z, et al. Highmobility group box-1 in ischemia-reperfusion injury of the heart. Circulation. 2008; 117:3216-26.

9. Yang X, Fullerton DA, Su X, Ao L, Cleveland JC Jr, Meng X. Pro-osteogenic phenotype of human aortic valve interstitial cells is associated with higher levels of toll-like receptors 2 and 4 and enhanced expression of bone morphogenetic protein 2. J Am Coll Cardiol. 2009;53:491-500.

10. Nair AR, Ebenezer PJ, Saini Y, Francis J. Angiotensin II-induced hypertensive renal inflammation is mediated through HMGB1-TLR4 signaling in rat tubuloepithelial cells. Exp Cell Res. 2015;335:238-47.

11. Jiang R, Cai J, Zhu Z, Chen D, Wang J, Wang Q, et al. Hypoxic trophoblast HMGB1 induces endothelial cell hyperpermeability via the TRL-4/caveolin-1 pathway. J Immunol. 2014;193:5000-12.

12. Gan LT, Van Rooyen DM, Koina ME, McCuskey RS, Teoh NC, Farrell GC. Hepatocyte free cholesterol lipotoxicity results from JNK1-mediated mitochondrial injury and is HMGB1 and TLR4-dependent. J Hepatol. 2014;61:1376-84.

13. Park JS, Svetkauskaite D, He QB, Kim JY, Strassheim D, Ishizaka A, et al. Involvement of toll-like receptors 2 and 4 in cellular activation by high mobility group box 1 protein. J Biol Chem. 2004;279:7370-7.

14. Yang HA, Hreggvidsdottir HS, Palmblad K, Wang HC, Ochani M, Li JH, et al. A critical cysteine is required for HMGB1 binding to Toll-like receptor 4 and activation of macrophage cytokine release. Proc Natl Acad Sci. 2010;107:11942-7.

15. Cai ZJ, Li F, Gong W, Liu WJ, Duan QL, Chen C, et al. Endoplasmic reticulum stress participates in aortic valve calcification in hypercholesterolemic animals. Arterioscler Thromb Vasc Biol. 2013;33:2345-54.

16. Li F, Zhao Z, Cai Z, Dong N, Liu Y. Oxidized low-density lipoprotein promotes osteoblastic differentiation of valvular interstitial cells through RAGE/MAPK. Cardiology. 2015;130:55-61.

17. Romani M, Rodman TC, Vidali G, Bustin M. Serological analysis of species specificity in the high mobility group chromosomal proteins. J Biol Chem. $1979 ; 254: 2918-22$.

18. Meng X, Ao L, Song Y, Babu A, Yang X, Wang M, et al. Expression of functional Toll-like receptors 2 and 4 in human aortic valve interstitial cells: potential roles in aortic valve inflammation and stenosis. Am J Physiol Cell Physiol. 2008;294: C29-35.

19. Zeng Q, Song R, Ao L, Weyant MJ, Lee J, Xu D, et al. Notch1 promotes the proosteogenic response of human aortic valve interstitial cells via modulation of ERK1/2 and nuclear factor-kappaB activation. Arterioscler Thromb Vasc Biol. 2013;33:1580-90.

20. Yang X, Meng X, Su X, Mauchley DC, Ao L, Cleveland JC Jr, et al. Bone morphogenic protein 2 induces Runx2 and osteopontin expression in human aortic valve interstitial cells: role of Smad1 and extracellular signal-regulated kinase 1/2. J Thorac Cardiovasc Surg. 2009;138:1008-15.

21. Zeng Q, Jin C, Ao L, Cleveland JC Jr, Song R, Xu D, et al. Cross-talk between the Toll-like receptor 4 and Notch1 pathways augments the inflammatory response in the interstitial cells of stenotic human aortic valves. Circulation. 2012;126: S222-30.
22. Callegari A, Coons ML, Ricks JL, Rosenfeld ME, Scatena M. Increased calcification in osteoprotegerin-deficient smooth muscle cells: dependence on receptor activator of NF-kappaB ligand and interleukin 6. J Vasc Res. 2014;51:118-31.

23. Speidl WS, Cimmino G, Ibanez B, Elmariah S, Hutter R, Garcia MJ, et al. Recombinant apolipoprotein A-I Milano rapidly reverses aortic valve stenosis and decreases leaflet inflammation in an experimental rabbit model. Eur Heart J. 2010;31:2049-57.

24. Al-Aly Z, Shao JS, Lai CF, Huang E, Cai J, Behrmann A, et al. Aortic Msx2-Wnt calcification cascade is regulated by TNF-alpha-dependent signals in diabetic Ldlr-/- mice. Arterioscler Thromb Vasc Biol. 2007;27:2589-96.

25. Yu Z, Seya K, Daitoku K, Motomura S, Fukuda I, Furukawa K. Tumor necrosis factor-alpha accelerates the calcification of human aortic valve interstitial cells obtained from patients with calcific aortic valve stenosis via the BMP2-Dlx5 pathway. J Pharmacol Exp Ther. 2011;337:16-23.

26. Glushkova OV, Khrenov MO, Novoselova TV, Lunin SM, Parfenyuk SB, Alekseev SI, et al. The role of the NF-kappaB, SAPK/JNK, and TLR4 signalling pathways in the responses of RAW 264.7 cells to extremely low-intensity microwaves. Int J Radiat Biol. 2015;91:321-8.

27. Nguyen CT, Kim EH, Luong TT, Pyo S, Rhee DK. TLR4 mediates pneumolysininduced ATF3 expression through the JNK/p38 pathway in Streptococcus pneumoniae-infected RAW 264.7 cells. Mol Cells. 2015;38:58-64.

28. Byun EB, Sung NY, Park JN, Yang MS, Park SH, Byun EH. Gamma-irradiated resveratrol negatively regulates LPS-induced MAPK and NF-kappa B signaling through TLR4 in macrophages. Int Immunopharmacol. 2015;25: 249-59.

29. Su X, Ao L, Shi Y, Johnson TR, Fullerton DA, Meng X. Oxidized low density lipoprotein induces bone morphogenetic protein-2 in coronary artery endothelial cells via Toll-like receptors 2 and 4. J Biol Chem. 2011;286:12213-20.

30. Song R, Zeng QC, Ao LH, Yu JA, Cleveland JC, Zhao KS, et al. Biglycan induces the expression of osteogenic factors in human aortic valve interstitial cells via toll-like receptor-2. Arterioscler Thromb Vasc Biol. 2012;32:2711-20.

31. Wang Y, Shan J, Yang W, Zheng H, Xue S. High mobility group box 1 (HMGB1) mediates high-glucose-induced calcification in vascular smooth muscle cells of saphenous veins. Inflammation. 2013;36:1592-604.

32. Rajamannan NM. Atorvastatin attenuates bone loss and aortic valve atheroma in LDLR mice. Cardiology. 2015;132:11-5.

33. De Vecchis R, Di Biase G, Esposito C, Ciccarelli A, Cioppa C, Giasi A, et al. Statin use for nonrheumatic calcific aortic valve stenosis: a review with metaanalysis. J Cardiovasc Med (Hagerstown). 2013;14:559-67.

34. Panahi Y, Sahebkar A, Taghipour HR, Dadjou Y, Pishgoo B, Rakhshankhah AS. Atorvastatin therapy is not associated with slowing the progression of aortic stenosis: findings of a randomized controlled trial. Clin Lab. 2013;59: 299-305.

Key Words: aortic valve calcification, osteoblastic differentiation, HMGB1, TLR4 